\title{
Chauffage de pavillons par pompe à chaleur sol-eau sur tubes enterrés
}

\author{
Heating of detached houses \\ by soil-water heat pump with buried pipes
}

\author{
A. Menjoz \\ Responsable du Laboratoire Réservoirs et modélisations \\ Institut mixte de recherches géothermiques \\ (BRGM-AFME) - Orléans
}

L'énergie nécessaire au chauffage de l'habitat individuel
peut être distribuée à bas niveau thermique et extraite des
premiers mètres de sol par circulation d'un fluide calopor-
teur dans un système de tubes horizontaux mis en place dans
une tranchée. Ce système de captage en différé de l'énergie
solaire associe la pompe à chaleur et repose sur l'aptitude
du milieu naturel sollicité à se régénérer durant la période
estivale. Le suivi expérimental d'un tel système, réalisé
durant deux années par instrumentation in situ, permet d'en
analyser le comportement, les phénomènes physiques princi-
paux et certaines règles pratiques pour le dimensionnement.
The energy required for heating an individual home can be distributed at low thermal level and extracted from the first meters of soil by circulating a heat-carrying fluid through a system of horizontal pipes installed in a trench. This system for delayed collection of solar energy associates the heat pump and is based on the capacity of the solicited natural medium to regenerate during the summer period. The experimental monitoring of such a system conducted for two years by in situ instrumentation makes it possible to analyze the behavior, the main physical phenomena and certain practical rules for sizing.

\section{Introduction}

Le système de chauffage de l'habitat par pompe à chaleur sol-eau consiste à prélever une partie de l'énergie solaire emmagasinée par les premiers mètres de sol durant la période estivale, à l'aide d'un réseau de tubes enterrés à faible profondeur. Ce dispositif est particulièrement bien adapté aux habitations individuelles disposant d'une parcelle de taille moyenne, et pratiquement en tout lieu.

On sait en effet que le flux thermique apporté par le rayonnement solaire pénètre dans les couches superficielles du sous-sol par conduction notamment. Ce dernier phénomène physique, très variable selon la nature et la couverture du sol, a pour conséquence une variation continue et cyclique de la température avec la profondeur. "L'onde thermique " incidente naturelle est ainsi amortie avec la profondeur et déphasée dans le temps; la tempéra- ture est alors pratiquement constante vers $10-15 \mathrm{~m}$ en toute région, pour augmenter ensuite régulièrement sous l'influence du flux géothermique. Il est donc envisageable d'espérer récupérer une partie de ce stock énergétique naturel par la mise en place d'un échangeur enfoui à faible profondeur, et assurer la pérennité du prélèvement par un dimensionnement optimal du système. Ce système par pompe à chaleur sol-eau présente deux avantages :

- il est généralisable dans de très nombreuses régions à climat tempéré, et en l'absence de formations aquifères;

- la régénération thermique du volume exploité en hiver est assurée systématiquement et gratuitement en été grâce aux apports météoriques, pour autant que l'on sache maîtriser le dimensionnement et la puissance du système de captage thermique constitué par l'échangeur souterrain.

LA HOUILLE BLANCHE/No3/4-1985 
Plusieurs centaines d'exploitations de ce type fonctionnent actuellement en Europe (Suède, Suisse, France,.). Deux dispositifs sont proposés sur le marché et se distinguent par la profondeur moyenne et le mode d'enfouissement. Les tubes peuvent être mis en place en 2 ou
3 nappes horizontales par fouille d'une tranchée de 2 mètres de profondeur environ.

La seconde méthode fait appel à une excavation totale de la parcelle choisie sur $0.8 \mathrm{~m}$ (système à 1 nappe en général). Le remblaiement est ensuite effectué avec le matériau d'origine.

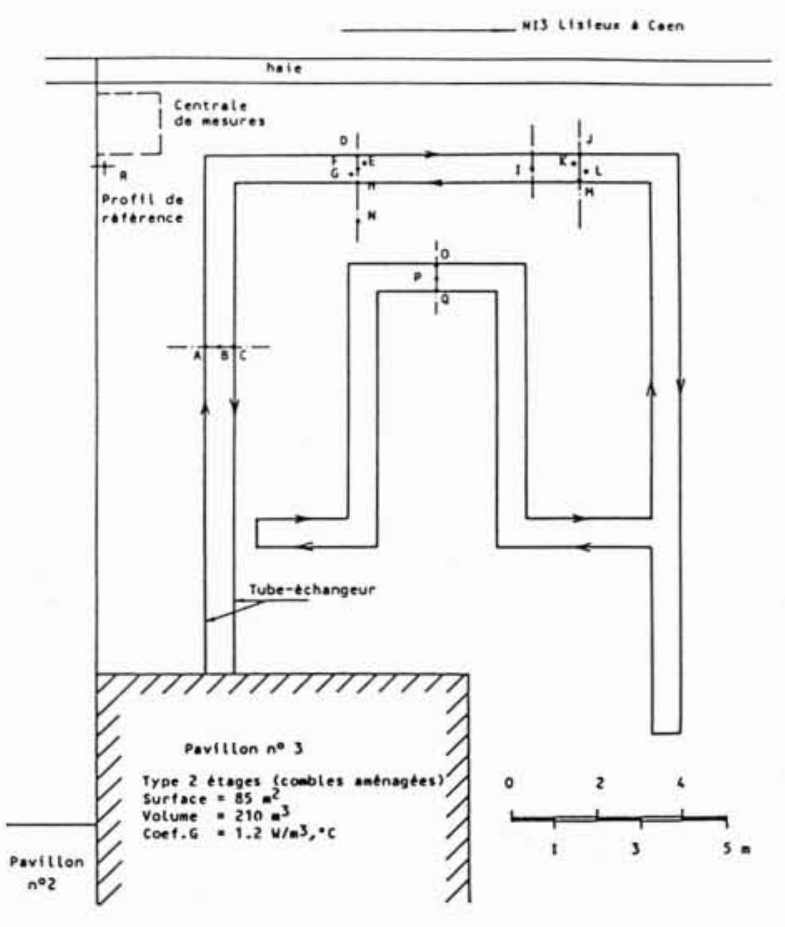

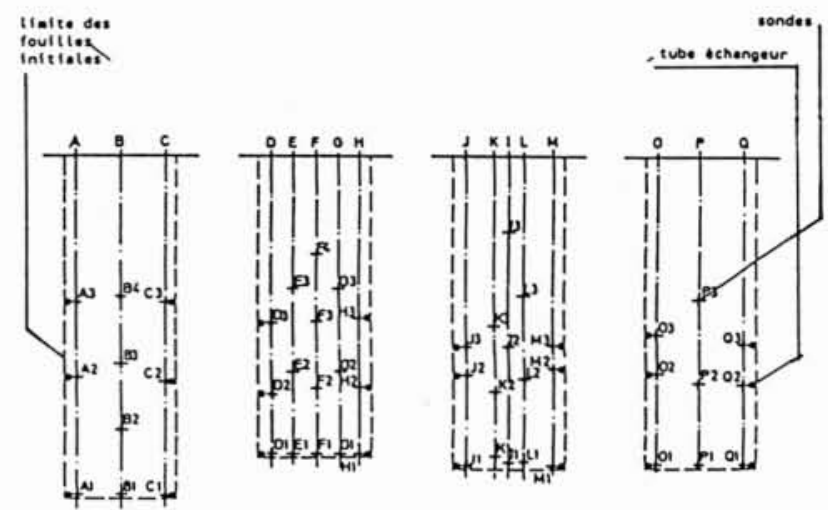

Figure 2. - Localisation des sondes et des tubes échangeurs dans les diverses sections transversales.

Figure 1. - Schéma d'implantation des stations de mesure sur la parcelle cadastrale affectée au pavillon n॰3.

\section{Contexte de \\ l'étude et description du système}

Le dispositif étudié est une réalisation en vraie grandeur (maître d'ouvrage : Office HLM), issue d'un concours national ayant pour thème le développement de nouveaux systèmes énergétiques pour l'habitat. Cette réalisation est constituée par le lotissement communal des «Petits Parcs ", situé à Crèvecœur-en-Auge dans le Calvados, et comprenant 7 pavillons équipés individuellement du même système de chauffage caractérisé par :

- une longueur donnée de tubes échangeurs enfouis en tranchée;

- une pompe à chaleur eau-eau;

- une distribution basse température par planchers chauffants;

- l'absence de système de chauffage d'appoint.

L'un des pavillons ( $\mathrm{n}^{\circ} 3$, figure 1$)$ a été sélectionné pour faire l'objet d'une investigation complète durant deux ans (1980-1982) : bilan énergétique et comportement thermique de la portion de sol sollicité par le système.

\subsection{Caractéristiques du site}

Sur une profondeur de $2,5 \mathrm{~m}$, le sol est constitué d'argiles fines avec quelques intercalations marneuses de faible épaisseur. Les interventions sur le site à diverses périodes de l'année et notamment en été ont permis de constater que le sol était en permanence saturé, hormis les 15 à 20 premiers $\mathrm{cm}$. Cette propriété caractéristique assure une capacité de régénération importante hors des périodes de prélèvement.

\section{2. Échangeurs tubulaires enterrés et instrumentation}

A chaque pavillon est attribuée une longueur de tranchée de $63 \mathrm{~m}$. Les tubes $(27 / 32)$ sont disposés en 3 nappes horizontales indépendantes, aux profondeurs respectives de $1,1.5$ et $2 \mathrm{~m}$. Soixante sondes thermiques ont été implantées in situ et raccordées à une centrale de mesure. Elles sont groupées en 18 stations $\mathrm{A}$ à $\mathrm{R}$ ( 3 à 4 sondes par 
verticale et 7 sondes pour le profil de référence). Les stations sont groupées en 4 sections transversales réparties sur la longueur totale de tranchée. Les sondes ont été construites spécialement pour cette application; une attention particulière a été apportée aux critères de fiabilité à long terme (étanchéité, thermistances de précision, blindage) et de positionnement dans l'espace vis-à-vis des tubes (figure 2).

\subsection{Système de chauffage et instrumentation}

La pompe à chaleur (CLIREF-EVE2.4519) a une puissance utile de $10 \mathrm{~kW}$ et un coefficient de performance théorique (COP) compris entre 3 et 4 . L'écart de température nominal au primaire est de $4{ }^{\circ} \mathrm{C}$ pour un débit de $1 \mathrm{~m}^{3} / \mathrm{h}$. La distribution par planchers chauffants est de type $35 / 30$ avec un débit de $0.6 \mathrm{~m}^{3} / \mathrm{h}$.

L'instrumentation disposée à l'intérieur comprend : 2 compteurs électriques jour/nuit, 2 compteurs à calories et volumétriques sur le primaire et le secondaire, ainsi qu'un compteur horaire sur chaque compresseur. L'ensemble des données disponibles permet donc de dresser le bilan thermique et d'évaluer le COP moyen saisonnier incluant tous les auxiliaires.

\section{Suivi expérimental : Résultats de deux années de mesures}

Les mesures périodiques ont débuté en août 1980, avant la première saison de chauffage avec une fréquence d'échantillonnage d'un relevé complet tous les 10 à 15 jours.

Le contrôle de l'homogénéité du milieu et de la fiabilité du système d'acquisition est effectué périodiquement (fin d'été par exemple) en traçant les corrélations températures-profondeurs et en examinant la dispersion autour du profil de référence (entre 0.25 et $0.5^{\circ} \mathrm{C}$ ). Cette méthode permet également de vérifier s'il subsiste une rémanence thermique (zones plus froides) en fin d'été.

L'évolution du profil de référence permet de quantifier l'amortissement des fluctuations thermiques de surface et l'effet de filtrage des diverses composantes du signal incident. Pour ce site, il existe ainsi une profondeur minimum de $1 \mathrm{~m}$, au delà de laquelle la température est périodique, reproductible, et par conséquent caractérisable par une relation théorique (calcul de dimensionnement pratiques). Ce profil, comme on le verra par la suite est exploité pour l'identification du signal de surface.

L'évolution des températures dans la zone perturbée est similaire quant à son allure à celle du profil de référence avec une dérive de quelques degrés durant les 7 mois de prélèvement, pour rejoindre ensuite plus rapidement l'évolution naturelle. Les diverses sections transversales, choisies initialement pour examiner la variation longitudinale de température, ne montrent qu'un très faible écart le long des tubes. Le prélèvement thermique s'accompagne d'un refroidissement d'ensemble de tout le volume sollicité. Les températures les plus basses notées au voisinage des tubes ( $5 \mathrm{~cm}$ de l'axe) sont voisines de zéro, soit un écart maximal entre le fluide et le sol non perturbé de 6 à $7{ }^{\circ} \mathrm{C}$ (moyenne 3 à $4{ }^{\circ} \mathrm{C}$ ).

\subsection{Interprétation des résultats}

En raison de la faible perméabilité du milieu, les calculs théoriques et les vérifications a posteriori (analyse spectrale du signal naturel) montrent que l'apport énergétique dû à l'infiltration est très faible, soit quelques $\%$ et peut être négligé devant l'amplitude des transferts diffusifs. Le comportement thermique du milieu est alors fonction de deux conditions aux limites fondamentales : en surface et aux parois des tubes.

\subsection{Méthodes d'interprétation}

Le signal causal de surface est le paramètre-clé issu du bilan énergétique autour duquel s'articulent les profils micro-climatiques d'une part et thermique dans le sol d'autre part. On a choisi de tester ici une autre méthode reposant sur l'analyse spectrale du signal de référence. Cette méthode permet de généraliser la solution analytique connue du problème défini par une excitation de surface sinusoïdale monofréquence. Pour chaque fréquence identifiée, le logarithme de l'amplitude et le déphasage sont des fonctions linéaires de la profondeur.

Les signaux obtenus par valeurs discrètes, sont échantillonnés et filtrés par application d'une fonction "peigne ", puis soumis à une algorithme de transformation de Fourier numérique (FFT). Deux opérations sur les spectres sont très utiles: l'interspectre, lié à l'énergie mutuelle d'interaction entre deux signaux, et la fonction de cohérence analogue à un coefficient de corrélation pour chaque fréquence. Au plan pratique, on déduit de cette méthode les paramètres de la fonction de transfert et un nombre limité de basses fréquences telles que la cohérence soit proche de 1 afin de reconstituer un signal causal de surface dont les effets soient représentatifs dans la gamme de profondeurs des tubes-échangeurs (1 à $2.5 \mathrm{~m}$ ).

Connaissant les paramètres du sol et le signal de surface reconstitué, le comportement du sol en tout point est simulé par modèle bidimensionnel puis comparé aux mesures. Le modèle utilise la méthode des éléments finis (quadratiques) pour résoudre le problème de la diffusion thermique associé aux conditions aux limites variables en régime transitoire. Il est tout d'abord appliqué au profil naturel pour vérification du signal causal, puis aux diverses sections transversales où se situent les mesures. 


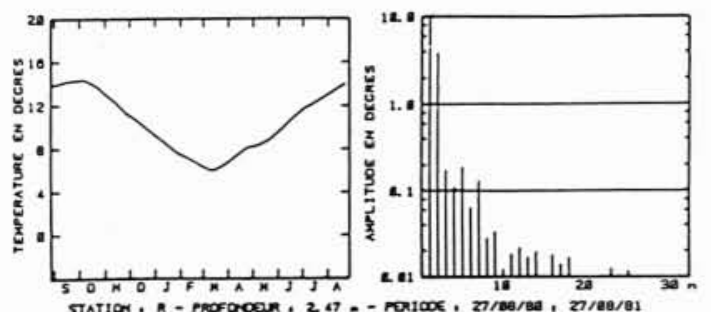

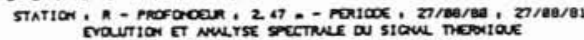

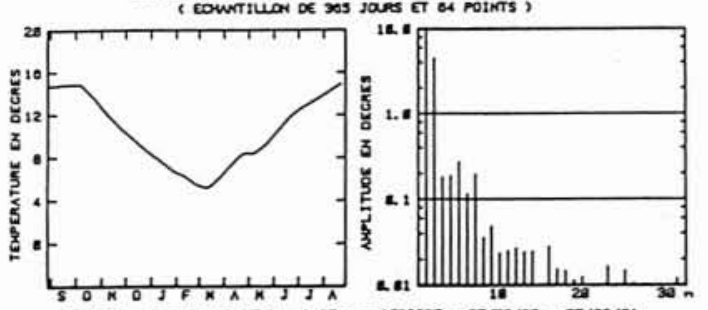

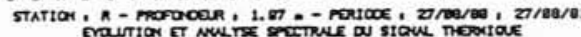

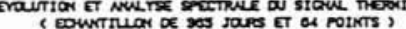

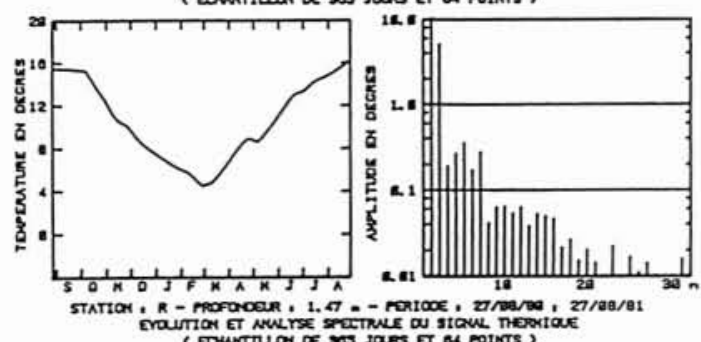
(a)

Figure 3
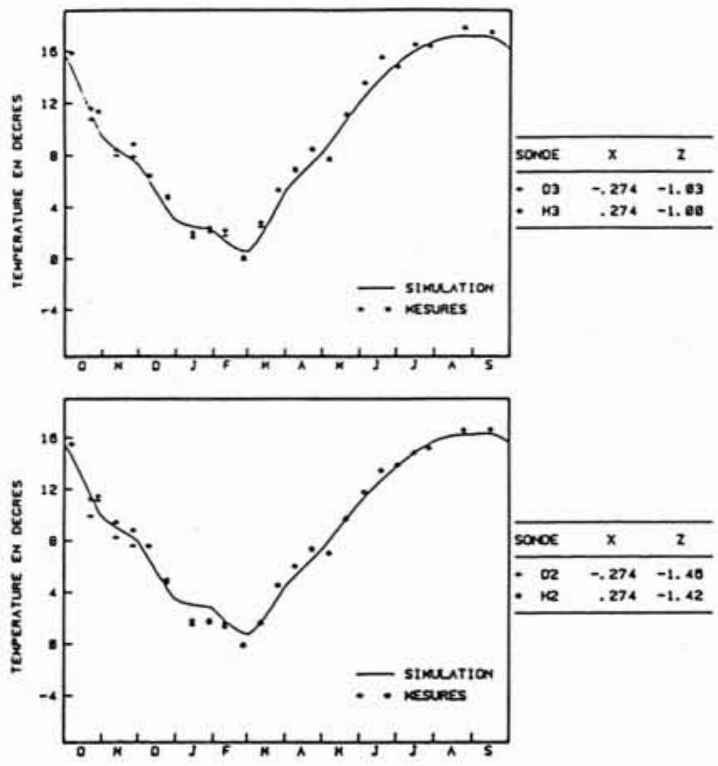

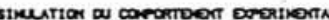

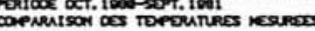

otmustrim nextac : an $6-0,20$
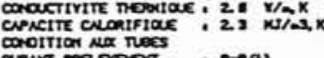

$: \ln _{p \rightarrow \infty}$

Figure 5
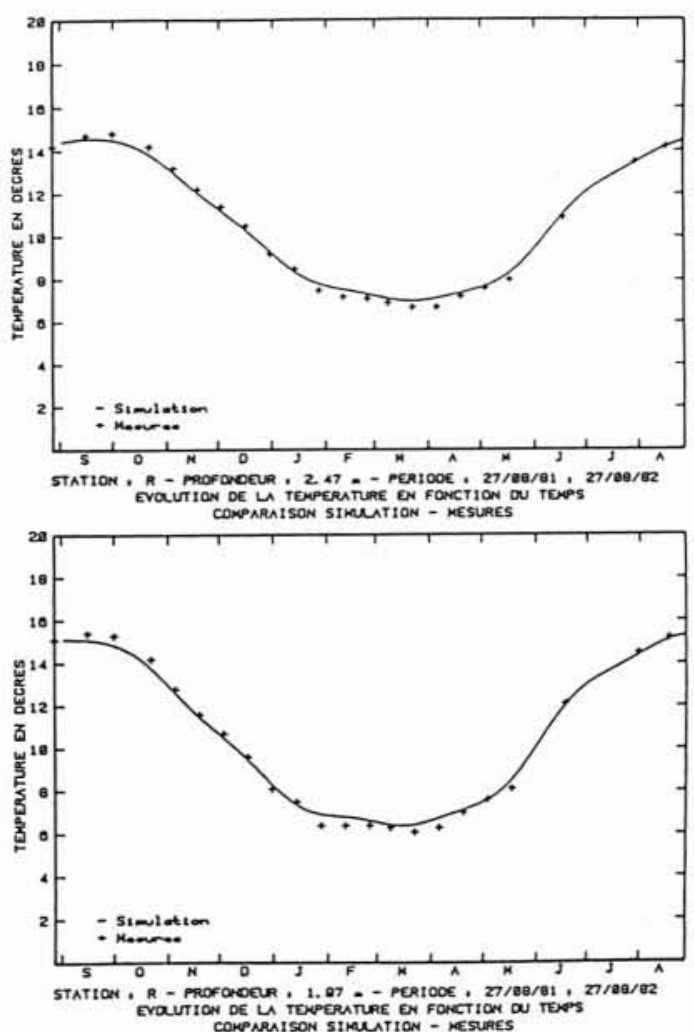

Figure 4
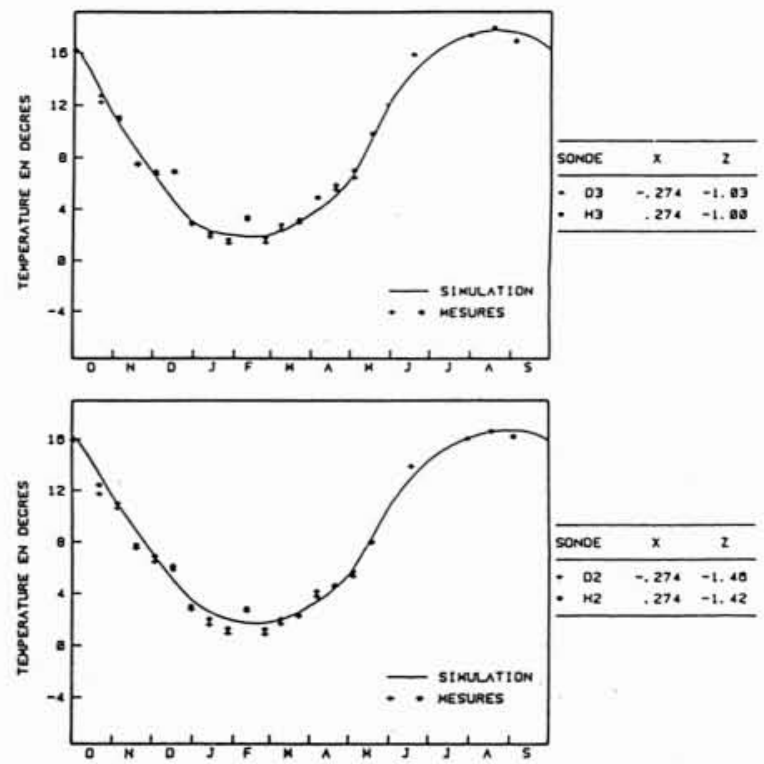

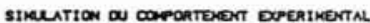
PERLOOE OCT. 10E1-SPPT. 1982 D conprentson

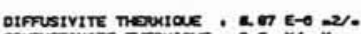

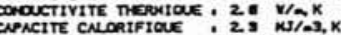
conortion Nux tueEs Hors PRE EVEGert 
Tableau I

Température moyenne annuelle déduite du profil de référence. (EchantilIon : 27.08.80 - 27.08.81)

\begin{tabular}{|c|c|}
\hline $\begin{array}{c}\text { VALEUR MOYENNE } \\
\text { TO ( C) }\end{array}$ & $\begin{array}{c}\text { PROFONDEUR } \\
\text { DE LA SONDE }\end{array}$ \\
\cline { 1 - 2 } 10.43 & $2.47 \mathrm{~m}$ \\
10.49 & $1.97 \mathrm{~m}$ \\
10.52 & $1.47 \mathrm{~m}$ \\
10.79 & $0.97 \mathrm{~m}$ \\
10.76 & $0.77 \mathrm{~m}$ \\
10.90 & $0.57 \mathrm{~m}$ \\
10.96 & $0.37 \mathrm{~m}$ \\
10.74 & air \\
\hline
\end{tabular}

Tableau II

Interprétation des signaux de référence par analyse des spectres. (Echantillon : $27.08 .80-27.08 .81)$

\begin{tabular}{|c|c|c|c|c|c|}
\hline \multirow{2}{*}{$\begin{array}{l}\text { RANG } \\
\text { HAR. }\end{array}$} & \multirow{2}{*}{$\begin{array}{l}\text { PERIODES } \\
\text { EN JOURS }\end{array}$} & \multicolumn{2}{|c|}{ D'APRES } & \multicolumn{2}{|c|}{ D'APRES } \\
\hline & & $\begin{array}{l}\text { SPECTRES } \\
\text { dT }\end{array}$ & $\begin{array}{l}\text { D'AMPLITUDE } \\
\text { Nb d'onde }\end{array}$ & $\begin{array}{l}\text { SPECTRES } \\
\text { phase }\end{array}$ & $\begin{array}{l}\text { DE PHASE } \\
\text { Nb.d'ondes }\end{array}$ \\
\hline $\begin{array}{l}1 \\
2 \\
3 \\
4 \\
5 \\
6\end{array}$ & $\begin{array}{r}365.0 \\
182.5 \\
121.7 \\
91.3 \\
73.0 \\
60.8\end{array}$ & $\begin{array}{l}8.7 \\
0.47 \\
0.6 \\
0.93 \\
0.5 \\
1.0\end{array}$ & $\begin{array}{l}0.330 \\
0.347 \\
0.366 \\
0.311 \\
0.353 \\
0.343\end{array}$ & $\begin{array}{r}-0.56 \\
1.02 \\
-0.32 \\
1.87 \\
-0.37 \\
3.25\end{array}$ & $\begin{array}{l}0.336 \\
0.378 \\
0.327 \\
0.320 \\
0.321 \\
0.329\end{array}$ \\
\hline
\end{tabular}

\subsection{Profil de référence ( $R$, figures 3 et 4)}

Les tableaux I et II présentent les moyennes annuelles déduites de l'examen de l'harmonique de rang 0 , et les 6 premières harmoniques déduites des spectres par régression linéaire et interspectres. Les diffusivités thermiques issues du nombre d'onde moyen annuel sont respectivement de 0.87 (1981) et $0.8 \mu \mathrm{m}^{2} / \mathrm{s}$ (1982). La conductivité thermique mesurée sur échantillon est de $1.9 \pm 0.1$ $\mathrm{W} / \mathrm{m}, \mathrm{K}$, d'où une capacité calorifique de $2.2 \mathrm{MJ} / \mathrm{m}^{3}, \mathrm{~K}$.

\subsection{Comportement aux voisinages des tubes}

La seconde condition aux limites nécessaires est traduite par un flux variable (processus itératif) à la paroi extérieure des tubes durant la période de chauffage, et par une condition adiabatique en été. Trois critères de contrôle sont utilisés :

- la température mesurée aux diverses sondes;

- l'énergie extraite du sol Ws déduite du COP saisonnier et des degrés-jours (DJU);

- l'énergie extraite mesurée entre chaque relevé de consommation (compteur à calories).

L'ajustement final obtenu se traduit par une erreur moyenne sur les 60 sondes de l'ordre de 0.5 à $0.7^{\circ} \mathrm{C}$ (figures 5 et 6). La puissance moyenne prélevée sur les tubes peut être approchée en pratique par une loi sinusoïdale de la forme :

$$
P(\mathrm{~W} / \mathrm{m})=P \max (1+\cos (w t+\sigma)) / 2
$$

avec $P$ max comprise entre 4 et $5 \mathrm{~W} / \mathrm{m}$. Le déphasage est déterminé à partir de l'analyse des températures extérieures dont on ne retient que l'harmonique de rang 1 . Ce type de conditions nécessite par contre la détermination de la conductivité thermique ajustée ici à sa valeur maximale mesurée sur échantillon saturé.

\subsection{Bilan énergétique}

Le bilan énergétique des 2 années, représenté à la figure 7 , se traduit par un coefficient de performance saisonnier avec auxiliaires de 2.1 la première année et 2 pour la seconde.

Cette valeur assez faible est due d'une part à la prise en compte des auxiliaires (circulateurs primaires et secondaires) et d'autre part à la basse température à l'évaporateur, de l'ordre de quelques degrés durant les mois les plus froids. 

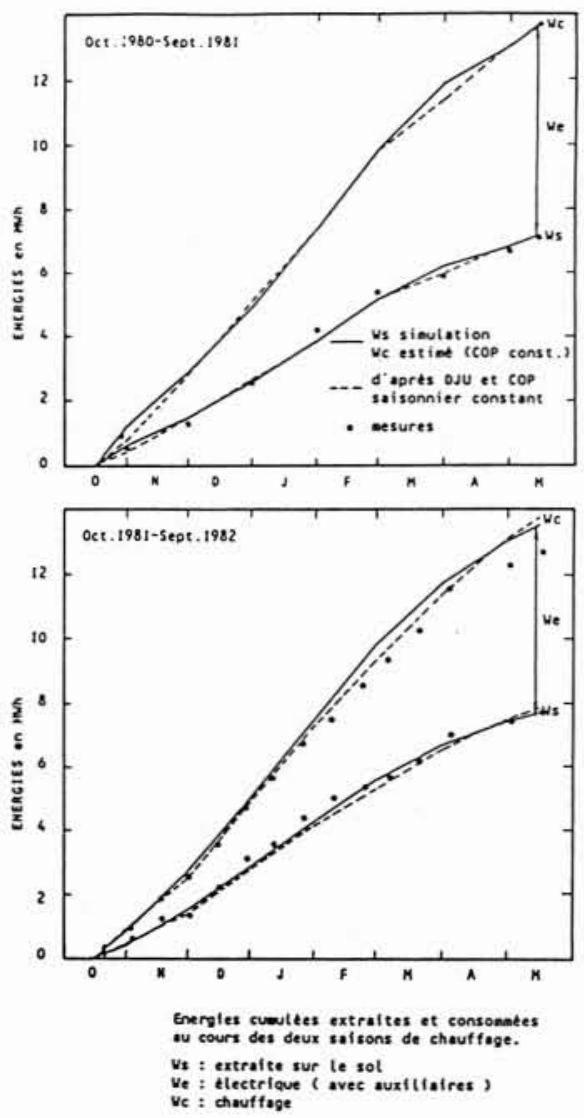

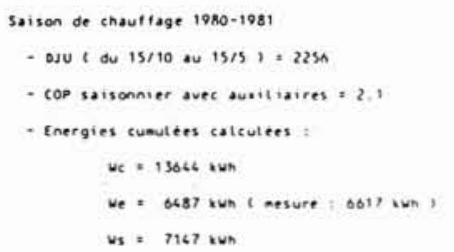

Saison de chauttage 1981-1982

- au c du 15/10 a 15/5) . 2272

- Cop saisonnier avec susitiaires - 1.90

- Energies cunultes calcultes :

$v_{c}=13761 \mathrm{kwh}$

ve $=5976 \mathrm{kwh}$

$\mathrm{ws}=7767 \mathrm{kwh}$ \& eesurt: $7706 \mathrm{kwh}$ ?

Figure 7

\section{4. Études théoriques de diverses variantes géométriques}

Le modèle numérique utilisé pour l'interprétation est ensuite exploité pour préciser l'influence du nombre, de la profondeur et de l'écartement des tubes sur le comportement du système et sous diverses conditions d'exploitation. La condition de surface retenue est réduite à l'harmonique annuelle, soit :

$$
T s=10.5+8.5 \cos (w t+\emptyset)
$$

La loi de prélèvement aux tubes est analogue à celle du $\S 3.4$ entre les dates du 15 octobre et du 15 mai; on se limite d'autre part à une température minimale du fluide à l'évaporateur $\left(-2^{\circ} \mathrm{C}\right)$.

\section{1. Échangeur à 1 nappe $(Z=1 \mathrm{~m})$}

L'incidence de l'écartement des tubes peut être examinée sur le tableau III, et montre qu'au plan énergétique, il est préférable d'utiliser un réseau à densité élevée avec une puissance de prélèvement faible; ce qui conduit à un refroidissement sensiblement homogène. Ce résultat est cohérent avec l'expérience des réalisations existantes.

\section{2. Échangeur à 2 nappes $(Z=1 \mathrm{~m}$ et $1.5 \mathrm{~m})$}

L'incidence de l'écartement des tranchées (largeur $0.7 \mathrm{~m}$ ) est résumée au tableau $I V$. Une part importante de l'énergie provient dans ce cas des couches de sol situées au dessous de la nappe inférieure.

\section{3. Échangeur à 3 nappes $(Z=1 \mathrm{~m}, 1.5 \mathrm{~m}$, et $2 \mathrm{~m})$}

Le prélèvement est pour ce cas plus homogène, mais un refroidissement résiduel de l'ordre de $1{ }^{\circ} \mathrm{C}$ peut subsister entre 3 et $4 \mathrm{~m}$ de profondeur (figures 8,9 et tableau $V$ ).

Compte tenu d'un léger surdimensionnement de sécurité, on peut retenir les règles pratiques suivantes quant aux longueurs de tubes ou de tranchées en mètres:

- pour le système à 1 nappe : longueur de tube égale à 4.5 à 5 fois la surface d'habitation $\left(\mathrm{en}^{2}\right)$;

- pour le système à 2 nappes: longueur de tranchées égale à 1.1 à 1.2 fois la surface à chauffer $\left(\mathrm{en}^{2}\right)$; - pour le système à 3 nappes: longueur de tranchées égale à 0.75 à 0.8 fois la surface à chauffer $\left(e n \mathrm{~m}^{2}\right)$. 


\begin{tabular}{|c|c|c|c|c|c|}
\hline $\begin{array}{c}\text { ECARTEMENT } \\
\text { DES TUBES } \\
\text { EN m }\end{array}$ & $\begin{array}{c}\text { ENERGIE } \\
\text { EXTRAITE } \\
\mathrm{kWh} / \mathrm{m}\end{array}$ & PMAX & $\begin{array}{c}\text { LONGUEUR } \\
\text { DE TUBES } \\
\text { EN m }\end{array}$ & $\begin{array}{c}\text { ENERGIE } \\
\text { EXTRAITE } \\
\text { WS (kWh) }\end{array}$ & $\begin{array}{c}\text { CHAUFFAGE } \\
\text { (cOP=2.5) } \\
\text { WC (kWh) }\end{array}$ \\
\hline 0.5 & 20.60 & 5.3 & 799 & 16459 & 27432 \\
1. & 30.32 & 7.8 & 399 & 12098 & 20163 \\
2. & 37.32 & 9.6 & 198 & 7389 & 12315 \\
\hline
\end{tabular}

Tableau III

Incidence de l'écartement des tubes pour un système à 1 nappe. (Emprise au sol $20 \mathrm{~m} \times 20 \mathrm{~m}$ - Fonctionnement hors gel)

\begin{tabular}{|c|c|c|c|c|c|}
\hline $\begin{array}{c}\text { ECARTEMENT } \\
\text { DES TUBES } \\
\text { EN } \mathrm{m}\end{array}$ & $\begin{array}{c}\text { ENERGIE } \\
\text { EXTRAITE } \\
\mathrm{kWh} / \mathrm{m}\end{array}$ & PMAX & $\begin{array}{c}\text { LONGUEUR } \\
\text { DE TUBES } \\
\text { EN m }\end{array}$ & $\begin{array}{c}\text { ENERGIE } \\
\text { EXTRAITE } \\
\text { WS (kWh) }\end{array}$ & $\begin{array}{l}\text { CHAUFFAGE } \\
\text { (COP=2.5) } \\
\text { WC (kWh) }\end{array}$ \\
\hline 2. & 23.32 & 6.0 & 555 & 12944 & 21573 \\
3. & 25.27 & 6.5 & 393 & 9929 & 16548 \\
\hline
\end{tabular}

Tableau IV

Incidence de l'écartement des tranchées pour un système à 2 nappes. (Emprise au sol $20 \mathrm{~m} \times 20 \mathrm{~m}-$ Fonctionnement hors gel)

\begin{tabular}{|c|c|c|c|c|c|}
\hline $\begin{array}{c}\text { ECARTEMENT } \\
\text { DES TUBES } \\
\text { EN } \mathrm{m}\end{array}$ & $\begin{array}{l}\text { ENERGIE } \\
\text { EXTRAITE } \\
\mathrm{kWh} / \mathrm{m}\end{array}$ & $\begin{array}{l}\text { PMAX } \\
W / m\end{array}$ & $\begin{array}{c}\text { LONGUEUR } \\
\text { DE TUBES } \\
\text { EN m }\end{array}$ & $\begin{array}{l}\text { ENERGIE } \\
\text { EXTRAITE } \\
\text { WS (kWh) }\end{array}$ & $\begin{array}{l}\text { CHAUFFAGE } \\
(C O P=2.5) \\
\text { WC }(k W h)\end{array}$ \\
\hline 3. & 20.99 & 5.4 & 589 & 12363 & 20605 \\
\hline 4. & 22.16 & 5.7 & 466 & 10325 & $17 \quad 208$ \\
\hline
\end{tabular}

Tableau V

Incidence de l'écartement des tranchées pour un système à 3 nappes. (Emprise au sol $20 \mathrm{~m} \times 20 \mathrm{~m}-$ Fonctionnement hors gel)

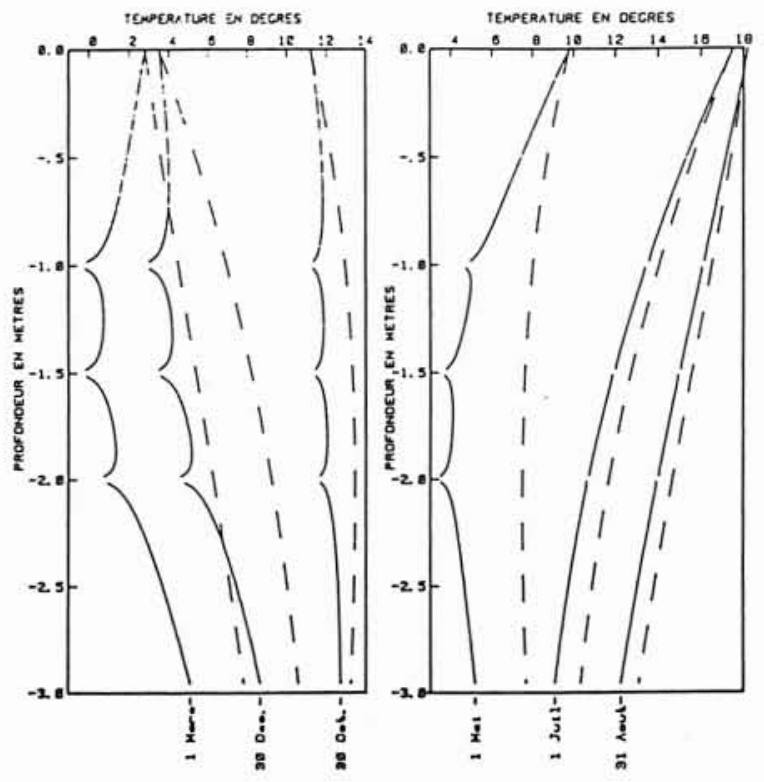

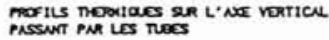

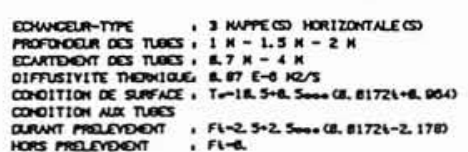

Figure 8

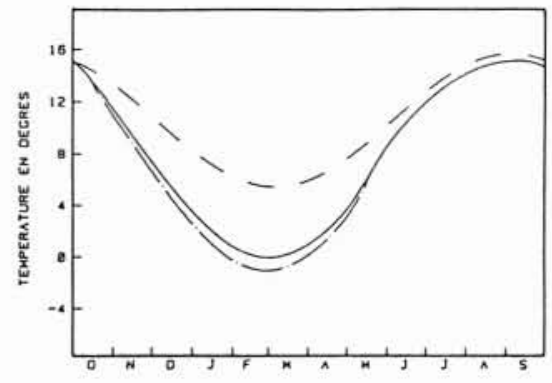

$2.1,5$
Veitinage

c.. in..

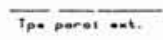

T.mp. notur.1:-

T.epp. du fluide

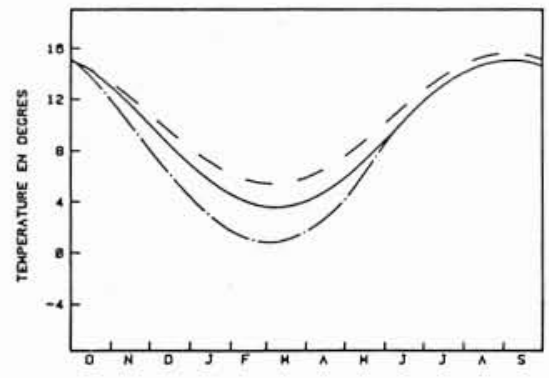

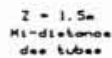

entre tromohic.:

dane iranohi.

T.op. notwan:11:-

EVOUTION DES TEMPERATURES DE OIVERS

POINTS AU COURS OU TENPS

ECUNCEUR-TYPE 3 MAPPE (S) MORIZONTALE (S)

PROFONOEUR DES TUEES : $1 M-1,5 N-2$

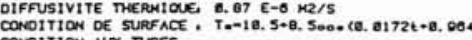

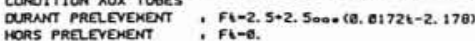

Figure 9 


\section{Conclusions}

Les diverses approches par modèles de simulation ont permis de reproduire fidèlement le comportement du sol durant les deux années de suivi expérimental :

- L'ajustement simulation-mesures a pu être réalisé à l'aide de paramètres constants annuels et du signal causal de surface déduits de l'analyse d'un profil de référence. - Pour le site étudié, caractérisé par une argile saturée, les résultats confirment l'hypothèse initiale quant à l'influence prépondérante des phénomènes de diffusion thermique.

- Le fonctionnement d'un système de captage à plusieurs nappes horizontales peut être traduit avec une bonne approximation en considérant une puissance de prélèvement linéique variable dans le temps, mais identique à un instant donné pour les différentes nappes. Cette puissance peut être déduite de manière simple à partir des données climatiques locales et en considérant un coefficient de performance moyen saisonnier.

- La fiabilité des calculs de simulation dépend pour une large part de la détermination de la température de surface qui constitue le signal causal essentiel. Dans ce sens, la méthode par analyse spectrale est particulièrement intéressante et peu coûteuse; sa mise en œuvre n'implique que l'implantation et le relevé périodique de quelques sondes sur une verticale de référence.
- Une puissance d'extraction maximale de 5 à $6 \mathrm{~W} / \mathrm{m}$, pour un système à 3 nappes, permet un fonctionnement moyen hors gel et une régénération naturelle complète entre les périodes de chauffage.

Le système à 3 nappes examiné dans cette étude présente des performances similaires à celles du système à 2 nappes. Il permet toutefois une certaine souplesse de gestion au plan du mode de prélèvement, un refroidissement plus homogène sur un plus grand volume, et par conséquent pour une même énergie prélevée, une régénération plus rapide durant les périodes d'arrêt.

\section{Remerciements}

Cette étude a été réalisée grâce à l'appui technique et financier des organismes publics (Ministère de l'Environnement et du Cadre de Vie, Mission Energie et Bâtiment, Plan Construction, Commissariat à l'Energie Solaire, EDF, CNET-HLM, BRGM) et en collaboration avec le Service Géologique Régional Basse Normandie du BRGM (MM. Dassibat, Matheron, Pascaud) et le CNET-HLM de Caen (MM. Delacour, Lauzanne, Mairesse).
M. Clément. Pouvez-vous donner quelques renseignements sur l'économie du procédé?

M. MENJOZ. - Par rapport au chauffage électrique intégré le surcoût est d'environ $30000 \mathrm{~F}$. en francs 1979 et l'économie réalisée de l'ordre de $2700 \mathrm{~F}$. par an. Le temps de retour est donc relativement long.

Le facteur pénalisant est la faible température du fluide caloporteur durant plusieurs mois de l'année, d'où un effondrement du coefficient de performance. Une régénération de la source froide durant l'été améliorerait ce coefficient dans la mesure où l'on parerait aux pertes thermiques à travers la surface du sol par un isolant. Avec des planchers chauffants on pourra par exemple faire circuler le fluide en court-circuitant la pompe à chaleur, créant ainsi une certaine climatisation.

M. SAUTY. - La modélisation par série de Fourier dont les paramètres ont été identifiés à partir de mesures sur le site de Crèvecœur est-elle directement exploitable pour prévoir le fonctionnement d'une autre installation dans un sol de caractéristiques thermiques différentes et pour une autre géométrie d'implantation de capteurs?

M. MENJOZ. - Jusqu'alors des règles empiriques de dimensionnement des installations ont été utilisées qui se sont révélées parfaitement adaptées.
L'étude montre qu'on peut se passer de mesures fines telles que celles de micrométéorologie de surface, de flux, etc. et que la connaissance du signal causal de surface et des paramètres du sol, acquise au moyen de relevés de température sur deux mètres de profondeur durant six mois ou un an permet une utilisation du modèle dans d'autres conditions.

Il y a toujours un léger surdimensionnement de sécurité et le fluide caloporteur contient une certaine proportion de glycol pour passer certains pointes occasionnelles.

M. SAUTY. - Donc le modèle est adaptable à d'autres conditions que celles de l'expérimentation sous réserve de procéder à quelques mesures spécifiques sur le nouveau site.

M. GOGUEL - Vous avez montré un diagramme indiquant les apports en chaleur de l'électricité : pompe à chaleur et électricité auxiliaire, donc finalement effet Joule, puisque les pertes des auxiliaires se transforment en élévation de température sur le fluide de chauffage. En bas du diagramme, vous avez la chaleur extraite du sol.

Je souhaiterais voir ce même diagramme exprimé en exergie.

L'exergie dépend des températures de référence. Or, si l'on prend une température de référence très semblable, on s'apercevra que la chaleur apportée au sol n'entraîne pas d'exergie, mais qu'on utilise beaucoup mieux la valeur énergétique du courant électrique. 
Je crois que le plaisir de voir ce diagramme un peu déformé ne justifie pas l'emploi de l'exergie. Mais si l'on devait comparer avec la pompe à chaleur sur air, on aurait des températures différentes, et c'est par l'intermédiaire de l'exergie qu'avec la même température de référence la comparaison se ferait le mieux.

M. TASTAVI. - En réponse à la question de M. Clement concernant l'économie du procédé on peut dire à partir de plusieurs exemples autour de Lausanne :

(1) Sur plusieurs années d'exploitation d'une PAC eau/eau utilisant le sol comme source froide, nous avons constaté qu'il n'y avait pas de refroidissement du sol. Le tableau suivant donne les consommations d'électricité et le coût pour trois saisons de chauffage.

\begin{tabular}{|l|c|c|c|c|c|}
\hline \multirow{2}{*}{ Saison } & \multirow{2}{*}{$\mathrm{kWh}$} & \multirow{2}{*}{ Degrés jours } & $\mathrm{kWh}$ & \multirow{2}{*}{ SFr. } & \multirow{2}{*}{$\mathrm{Fr} . \mathrm{Fr}$. } \\
\cline { 4 - 4 } & & & Degrés jours & & \\
\hline $1980-81$ & $5^{\prime} 346$ & $3^{\prime} 132$ & 1,71 & $572 .-$ & $2^{\prime} 120 .-$ \\
$1981-82$ & $4^{\prime} 317$ & $2^{\prime} 963$ & 1,46 & $462 .-$ & $1711 .-$ \\
$1982-83$ & $3^{\prime} 729$ & $2^{\prime} 903$ & 1,28 & $399 .-$ & $1^{\prime} 478 .-$ \\
\hline
\end{tabular}

(2) En ce qui concerne le temps de retour (durée de l'amortissement du capital supplémentaire investi pour une PAC par rapport à une chaudière à mazout), les résultats obtenus sur plusieurs installations aux environs de Lausanne, donnent une valeur moyenne de cinq ans. (Coût du courant électrique : $11 \mathrm{ct}$ suisses/kWh).

Pouvez-vous dire quels sont les surcoûts lorsque la chaleur est prise sur l'air.?

M. MENJOZ. - Je n'ai pas fait la comparaison. 11 m'est difficile de vous répondre.
M. FAVROD. - Quelle est la durée de gel qui pourrait arrêter l'exploitation?

M. MENJOZ - Dans une région au climat tempéré comme le Calvados, laddition de glycol dans le fluide caloporteur permet à l'exploitation thermique de se poursuivre durant des périodes de gel courantes de l'ordre de quelques jours. L'exploitation peut très bien se poursuivre sur sol gelé.

M. FAVROD. - Ce système peut-il remplacer un autre système ou nécessite-t-il un chauffage d'appoint?

M. MENJOZ. - Dans la région Ouest de la France où les températures moyennes les plus basses sont de 10 environ ce systeme peut fonctionner sans appoint. Dans les autres régions il est prudent de prévoir un chauffage d'appoint.

M. MARCHAL (CETIAT). - Avez-vous étudié le diamètre optimal à donner aux tubes de l'échangeur enterré en raison de l'importance des pertes de charge?

M. MENJOZ. - Le choix de la nature et de la dimension des tubes résulte de contraintes technologiques et physiques. Les problèmes de corrosion ont conditionné la nature des tubes (P.V.C.) et le choix d'un diamètre intérieur de $27 \mathrm{~mm}$ est le résulat d'études empiriques effectuées par le promoteur. Ce choix s'est révélé satisfaisant car la perte de charge n'a été que de I bar sur les $380 \mathrm{~m}$ de tubes.

M. SOURIEAU. - Quelle est l'importance de la couverture? Faut-il une préparation du terrain pour favoriser l'arrivée du rayonnement solaire?

M. MENJOZ. - En principe le site ne doit être ni cultivé, ni ombragé. La couverture était ici une pelouse et les bâtiments suffisamment bas pour ne pas créer de zone d'ombre importante.

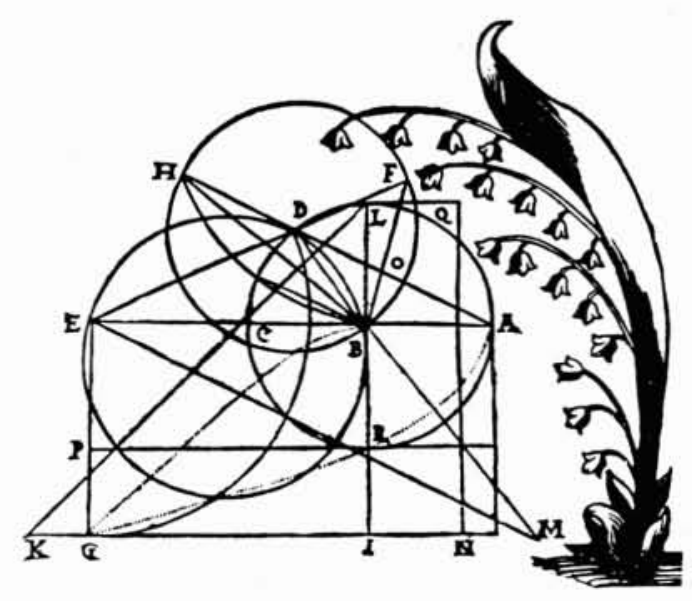




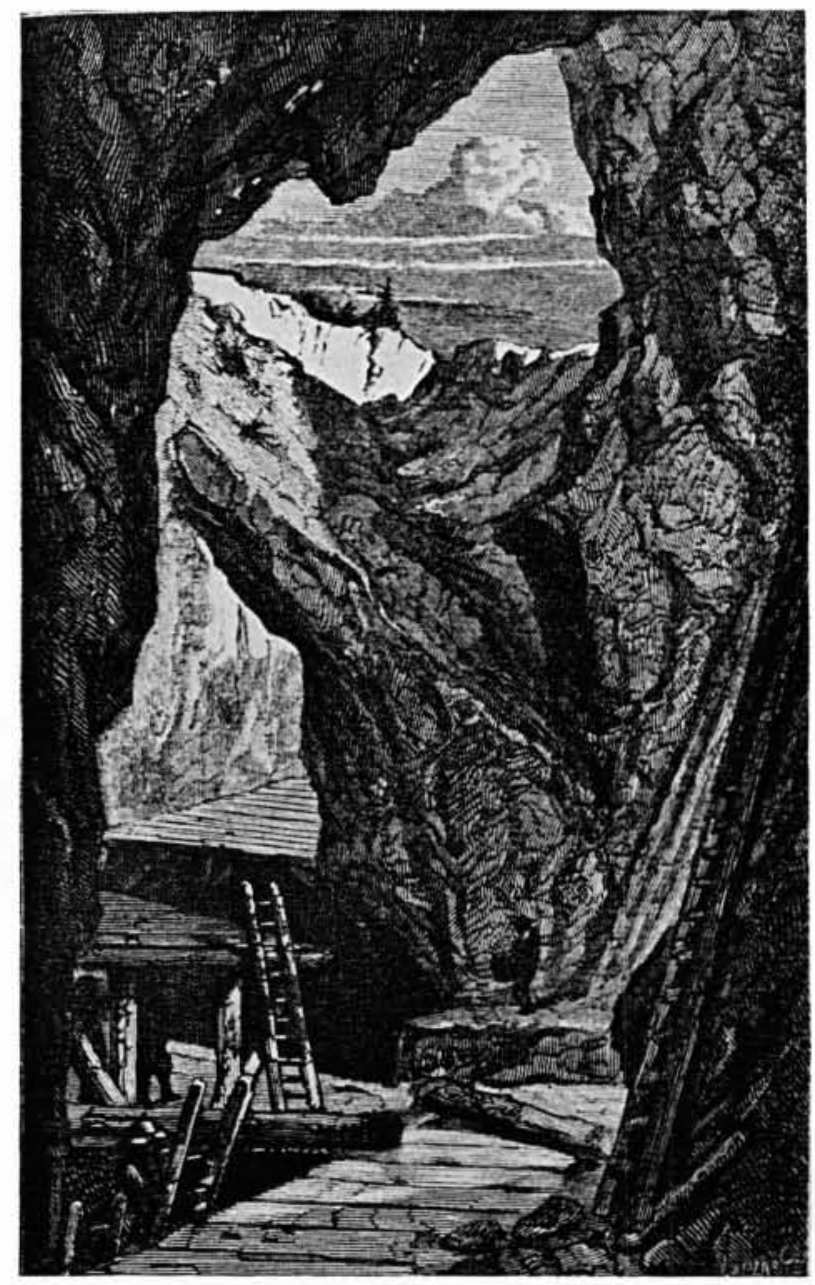

